\title{
NUTRITIONAL DEFICIENCIES: A REVIEW
}

\author{
Aleena s kumar \\ Amity Institute of Biotechnology \\ Amity University, Noida, Uttar Pradesh, India
}

\begin{abstract}
Nutrients like proteins, vitamins, minerals, carbohydrates and lipids are required for the right function of our body. Nutritional deficiency disorders are a major problem faced by the world, millions of people in the world suffer from undernourishment. Deficiency occurs when the food we are consuming does not contain the required amount of nutrients or when our body is not being able to absorb nutrients due to some genetic disorders or other secondary reasons. Nutritional deficiency is considered as the 'hidden hunger' and a person can suffer from deficiency of vitamins, proteins, minerals, carbohydrates and fatty acids. This review describes the different types of nutritional deficiencies, their causes, symptoms, treatment and prevention. The deficiencies are mostly asymptomatic but in a long time can very adversely affect the body functions and impair the immune system and cause chronic illness and infections. The severity of symptoms and the treatment varies from person to person. Poverty and low health literacy are the most important reasons for the prevalence of nutritional deficiencies in the world. However, these deficiencies can be prevented by promoting health education among adults, encouraging a healthy and active lifestyle and providing nutritional and healthy food to the ones who do not have access to it. Nutrition campaigns, supplementation and genetic engineering of plants and food crops has decreased the pervasiveness of deficiency disorders.
\end{abstract}

Keywords - Malnutrition, vitamin deficiency, mineral deficiency

\section{INTRODUCTION}

Nutrients are required for the metabolic reactions, repairing of tissues and growth, producing hereditary substances and overall development of a human body. Nutrients can be grouped into macronutrients and micronutrients. Macronutrients are required in large amounts and also provide a great amount of energy. These are carbohydrates, protein and fats. The micronutrients like vitamins and minerals are required in lesser amounts and majorly supports the metabolic reactions. Many of the nutrients are very important for the proper functioning of the body and cannot be synthesised within the human body, these nutrients are called essential nutrients. There are 9 amino acids, 2 fatty acids, 13 vitamins and 15 minerals in this category. Our body receives these nutrients through a well-balanced diet. (1))
When an adequate amount of these nutrients is not received by the body it leads to nutritional deficiency. A short period of dietary deficiency can be handled by the body but when the deficiency prolongs it leads to many developmental abnormalities, diseases and disorders. The major causes of nutritional deficiency are using highly refined food, consuming starchy fruits, vegetables and food grown in mineral deficient soil and also a large amount of nutrients is lost due to over cooking.

Generally nutritional deficiency occurs in two forms:

i. Primary deficiency occurs due to inadequate intake of nutrients. It can be divided into major deficiencies that cause complications related to the major organ systems and functions such as heart, metabolism in cell, vision, overall body development and growth. The minor deficiencies cause irritations like lesions, muscle and joint weakness, hair, teeth and nail problems.

ii. secondary deficiency occurs when the body is not able to absorb the nutrients due to gastrointestinal tract dysfunction causing altered appetite and indigestion or by metabolic abnormalities. Interference by other nutrients, increased loss of other nutrients, increased excretion, smoking, alcohol and even pollution are some of the secondary causes of deficiency diseases.

There are mainly three types of nutrient deficiencies Protein energy malnutrition (PEM) or protein calorie malnutrition, vitamin deficiency and mineral deficiency.

\section{PROTEIN ENERGY MALNUTRITION}

Protein energy malnutrition or protein calorie malnutrition occurs when there is inadequate intake of proteins and nonprotein energy sources such as carbohydrates and fats and affects the whole body. Proteins are present in every cell of the human body. It is necessary for the growth and maintenance, structure and every activity taking place inside the human body needs protein in one or the other form. The major sources of protein are milk, egg, meat, fish, pulses and cereals etc. carbohydrates provide energy to the cells and consuming carbohydrate can cause decrease in the glycogen storage and cause increased production of keto acids in the body. This can lead to mineral loss from the bones, a functional and developmental problem of the central nervous system. fats are also a vital source of energy and helps with the absorption of vitamins such as A, E, D and $\mathrm{K}$ and also 
carotenoids. It is important for the functioning of proteins, epithelial cell functions, cells signalling pathways and are also a constituent of cell membrane. Fat, carbohydrate and protein can together lead to malnutrition and starvation but their increased level can have adverse effects on the body.

PEM is very common in developing countries. There are many factors that can lead to malnutrition in children and this includes social and economic factors, biological factors and environmental factors. PEM is very common among infants and young children who have a high level of nutritional Requirements. It also affects pregnant and lactating women which further affects the growth and nutritional status of the foetuses and infants. Aged people suffer from PEM mostly due to secondary reasons such as alteration in gastrointestinal systems. vegetarians are also at a risk of acquiring deficiency as some of the essential amino acids are scarcely found in the plant-based food products but this can be overcome by including a wide variety of plants in the diet. Protein energy malnutrition has many types, the primary forms are kwashiorkor, marasmus, kwashiorkor-marasmus and then the secondary forms like wasting disorder, alteration in gastrointestinal system etc.

\section{i. PRIMARY PROTEIN ENERGY MALNUTRITION}

\section{a. KWASHIORKOR}

Kwashiorkor, also known as wet, swollen or oedematous protein energy malnutrition is caused due to protein deficiency and is majorly seen in children between the age of 1 to 5 mainly due to premature abandonment of breastfeeding. The symptoms develop slowly over time. The abdomen protrudes due to weakening of abdominal muscles, distended intestine and enlarged liver. The key symptoms are diarrhoea, enlarged liver, fatigue, frequent infection, irritation, muscle wasting, skin changes, including pigment loss or purple patches cracking or development of sores, slowed growth resulting in short stature, weight loss, rapid pulse rate and respiratory and breathing problems. Children suffering from kwashiorkor can also suffer from physical and mental disability and could be apathetic but irritable when held. Bodies affected with kwashiorkor fail to produce antibodies against diseases.

\section{b. MARASMUS}

Marasmus or dry primary energy malnutrition occurs due to deficiency in proteins, carbohydrates, fats and other nutrients and is a very serious problem. It occurs mainly in infants, chronic hunger, inadequate food supply, other vitamin deficiency can increase the danger of acquiring marasmus. The child will look thin and limbs appear as skin and bone due to loss of subcutaneous fat and severe muscle wasting. Pneumonia, malaria, meningitis and lactose intolerance can increase the risk of marasmus. The common symptoms are chronic diarrhoea, dry or wrinkled skin, loss of bowel or bladder controls, stunted growth, irritability, fretfulness and diminished intellectual abilities. Marasmus is more common than kwashiorkor.

\section{c. MARASMUS- KWASHIORKOR}

Marasmus-kwashiorkor is a combined form which occurs when the body is subjected to stressful conditions. This is often a life-threatening condition. The symptoms and treatment all depend upon which condition predominates, kwashiorkor or marasmus. In both the cases the cell mediated immunity is impaired thus increasing susceptibility to infections.

\section{ii. SECONDARY PROTEIN ENERGY MALNUTRITION}

Secondary PEM results from the following reasons:

1. Wasting disorder causes severe undernutrition like anorexia and edema which decreases appetite and disrupts the metabolism of nutrients.

2. Alteration in the gastrointestinal tract which interferes with digestion or absorption and even transportation of nutrients.

3. A person who underwent a major surgery, trauma or any critical illness can suffer from PEM as the energy and protein requirement of the body is too high.

Starvation is the most severe stage of deficiency of energy in the body and is the extreme form of protein energy malnutrition. The person becomes weak to even move and one by one their organs and the organ system starts failing and ultimately leading to death. Starvation is said to occur if the person loses more than $30 \%$ of their body weight due to undernourishment and if iIt exceeds $40 \%$ it could lead to death.

\section{VITAMIN DEFICIENCY}

Vitamins are essential micronutrients and they take part in various biochemical processes and have multiple functions. Vitamins are of two types fat soluble which includes vitamin A, D, E and K and fat soluble which are all types of vitamin B and vitamin $\mathrm{C}$. the main causes of vitamin deficiency are poverty, food faddism, inadequately supplemental prenatal feeding, alcoholism and malabsorption. Vitamin deficiency is common among all ages but mostly in children, pregnant or lactating women and even smokers and alcoholics as they need vitamins in high quantities. The effect of each type of vitamin deficiency changes with their function. Fat soluble vitamin deficiency takes more than one year to show but deficiencies due to water soluble vitamins takes just about a few weeks and months of undernutrition. Most commonly vitamin deficiency affects the central nervous system.

1. Vitamin A (Retinol): Its function is to maintain normal vision, reproduction, normal cartilaginous and bone growth and immunity against infections. Retinol 
deficiency causes night blindness, xerophthalmia, where irreversible drying of cornea occurs and abnormal epithelial cell growth. The younger the patient the more severe will be the disorders or the effects as the immune system is affected. Iron and zinc deficiencies may affect vitamin A level in the body. Vitamin A deficiency is rare and intake of vitamin A rich food such as yellow -orange coloured vegetables, milk, eggs, fish can prevent the occurrence of its deficiency

2. Vitamin B1 (Thiamine): Thiamine which is mostly acquired from whole grains, legumes, nuts and fish etc. helps with ATP production and function of central and peripheral nerve cells and the myocardium. The deficiency mainly occurs due to the use of refined carbohydrates. Its deficiency causes beriberi and in alcoholics causes Wernicke's and Korsakoff's syndrome. Thiamine deficiency causes dilation of heart, edema in legs and feet and even heart failure, also degeneration of nerves and cerebellum. Increased levels of glucose can worsen the conditions thiamine is required for its metabolism. Long term deficiency is life threatening.

3. Vitamin B2 (Riboflavin)also called the yellow enzyme is found in milk cheese, meats, eggs, legumes, wheatgerm, mushrooms and green leafy vegetables. It helps in red blood cells production and its deficiency also called as ariboflavinosis causes skin and mucosal lesions, cheilosis and also anaemia. The tongue may appear magenta in some cases. Eyes also become itchy and sensitive to light. Ariboflavinosis occurs together with deficiency of other nutrients.

4. Vitamin B3 (Niacin) is vital for cell metabolism. deficiency causes pellagra, Hartnup disease, a hereditary abnormality in metabolism of tryptophan, gastrointestinal symptoms such as burning sensation in larynx and pharynx, vomiting and constipation, central nervous system symptoms such as psychosis, impaired consciousness and dementia, also dandruff and dermatitis. Food rich in niacin are fish, poultry, whole grain, nuts and legumes. Nicotinamide is usually used in treatments of other diseases.

5. Vitamin B5 (Pantothenic acid) takes part in the fatty acid metabolism and synthesis of coenzyme A. Deficiency of pantothenic acid causes dermatitis, retarded growth, loss of hair, apathy, malaise, sleep deprivation, hypoglycemia and increased sensitivity to insulin, neurological symptoms such as numbness, staggering, muscle cramps and greying of hair. This is a rare condition, pantothenic acid is abundantly found in alfalfa, meat, milk, tomato, meat, honey etc.

6. Vitamin B6 (Pyridoxine) is vital for the metabolism of proteins, gene expression and neurotransmitter synthesis. It is abundantly found in most of the foods and hence deficiency is very rare. Protein energy malnutrition liver diseases, haemodialysis, increased level of alcohol and consumption of pyridoxine inactivating drugs can increase the risk of pyridoxine deficiency. Deficiency causes microcytic anaemia, depression, confusion, epileptiform convulsions, nausea, mental disorders, seizure in infants and retarded growth.

7. Vitamin B7 (biotin) functions as a coenzyme and its deficiency is mostly common among people who consume a lot of egg white as it contains protein which has antibacterial action called avidin which binds to biotin and prevents its absorption. Also, pregnant women, heavy smokers or drinkers and people with digestive disorders or people under parenteral nutrition for a long time can face deficiency of biotin. Biotin deficiency causes alopecia, poor growth, loss of muscular control, loss of muscular control, loss of appetite, weakness and hair fall. It also affects the lipid metabolism and immune system. tomatoes, onions, berries, banana, walnuts and meat are rich sources of biotin

8. Vitamin B10 (folic acid or folate) which is essential for the formation of red blood cells, development of foetal nervous system, promotes the growth of useful microorganisms within the bowel and also increases appetite. Green leaf and vegetables, yogurt, mushrooms, wheat germ and eggs are rich in folic acid but prolonged cooking could destroy it. Alcohol also interferes with folate absorption. Folic acid deficiency causes megaloblastic and macrocytic anaemia, diarrhoea, alopecia, depression confusion and deficiency during pregnancy causes foetal neural tube defects and other brain disorders.

9. Vitamin B12 (cobalamin) takes part in DNA synthesis and proper functioning of the neurological system, production of red blood cells and DNA. The dietary source of cobalamin are meats, poultry, milk and milk products. People who suffer from gastritis, anaemia, immune disorders, and who are vegetarians or vegans are most likely to develop cobalamin deficiency. Pernicious anaemia and degeneration of the spinal cord are some deficiency symptoms. Irritability, mild depression and paranoia can also occur in some cases. Neurological disorders, hypotonia, lethargy and withdrawal symptoms can be seen in infants.

10. Vitamin $C$ (Ascorbic acid) is essential for red blood cells, synthesis of collagen which keeps the tissues healthy, antibody production and also acts as an antioxidant. Vitamin $\mathrm{c}$ is found in citrus fruits, tomatoes, potatoes and strawberries. Its deficiency disrupts the synthesis of collagen hence affecting the tissues and thus causing coiled or splitting hair, joint effusions, edema, slow wound healing, easy bruising, bleeding gums or tooth loss and scurvy which is a connective tissue defect, and depresses the immune system and weakens the body.

11. Vitamin D (Calciferol) is synthesized within the body using sunlight but when the exposure to sunlight is less than deficiency due to food occurs.is important for calcium and phosphate absorption and also repair of bones. Direct 
ultraviolet B irradiation of the skin, fortified dairy products etc are sources of vitamin D. Calciferol deficiency is very common and affects the body's immune system. Deficiency of vitamin D causes rickets in children where inadequate mineralisation of bones occurs and affect the skeletal growth causing deformities, Osteomalacia in adults, osteoporosis when bones become thin and weak, depression, back and bone pain etc. people who live in the northern regions and who receives very less exposure to sunlight are at high risk.

12. Vitamin E (Tocopherol), also called fertility vitamin is very important for the functioning of the reproductive system and is a vital component of antioxidants. It is commonly seen among infants and people with impaired fat metabolism. The dietary supplements are wheat germ, green leafy vegetables and fats of vegetable origin. Deficiency of vitamin $\mathrm{E}$ in the early stages causes haemolysis and in prolonged and serious cases causes sterility, muscular dystrophy and increased haemolysis leading to macrocytic anaemia.

13. Vitamin $\mathrm{K}$ has three derivatives $\mathrm{K} 1, \mathrm{~K} 2$ and $\mathrm{K} 3$. It is important for the formation of blood-clotting factors and prevention of excess bleeding. These are acquired from leafy vegetables, wheat germ, etc. Vitamin K deficiency is very common among breastfed infants and causes hemorragic disease. The use of anticoagulant drugs such as warfarin can reduce the level of vitamin $\mathrm{K}$ in the cells.

\section{MINERAL DEFICIENCY}

Minerals are grouped into trace and major minerals depending upon the amount in which our body needs them. Major minerals are needed in about 0.1 gm per day and trace minerals are needed in $0.01 \mathrm{gm}$ per day. The most prominent deficiencies are iron, zinc and iodine. The cases where the body is deficient of a single nutrient is very rare, most of the minerals are dependent on each other and their deficiencies occur together. The minerals and their deficiencies are:

1. Calcium: calcium is very important for bone growth and strength, regulates the heart rate, lowers cholesterol and prevents muscle cramps. It occurs in a variety of food such as green, leafy vegetables, milk and eggs. Calcium deficiency occurs mainly due deficiency in vitamin $\mathrm{D}$ as it is vital to absorb dietary calcium. In women as age increases calcium loss also increases due to the reduction of hormone oestrogen in their bodies. The deficiency of calcium is called hypocalcemia. In the long term it could lead to osteoporosis, joint pain, brittle nails, high blood pressure and cholesterol, insomnia, tooth decay, nervousness, and similarly cognitive problems such as confusion, convulsions, depression and hyperactivity.
2. Potassium: potassium helps in maintaining a steady heart rate and prevents strokes. It is also important for the health of the nervous system and maintaining fluid balance in our body. Potassium deficiency also known as hypokalemia, is generally related with sodium deficiency. Deficiency can be caused by excessive loss of body fluids through chronic vomiting, diarrhoea, excessive sweating and also due to blood loss. Potassium regulates muscle contractions hence its deficiency could lead to muscle cramps, spasms, weakness, fatigue, breathing difficulties, bloating and constipation. Severe cases can result in cardiac arrhythmias, muscular stiffness which is due to rapid muscle breakdown and also affect nerve function. Potassium rich food are fruits such as banana, avocado, vegetables, beans and nuts.

3. Magnesium: magnesium is very important for the effective utilisation of calcium and potassium. It also takes part in enzyme reactions to produce energy. Causes include alcoholism, medications, diarrhoea, urinary loss and even diabetes mellitus. Magnesium deficiency can cause faulty transmission of nerve and muscle impulses, confusion, irregular heart rate, seizures, cardiac arrest, asthma, insomnia and irritable bowel syndrome and in severe cases potassium and calcium absorption could be affected. The dietary sources are salmon, soymilk, cashew, spinach, banana, and nuts such as peanuts, etc.

4. Sodium: sodium is an electrolyte and helps with the regulation of water in the body. Sodium deficiency also known as hyponatremia is caused due to dehydration or drinking too much water, liver and kidney failure and by syndrome of inappropriate antidiuretic hormone (SIADH). Symptoms are weakness, muscle cramps, headache, nausea and in severe cases loss of consciousness seizures and ultimately even coma.

5. Phosphorous: phosphorous maintains and helps with the growth of bones and teeth, supports cell growth and is vital for the absorption and utilisation of vitamins. Phosphorous deficiency is rare as it is abundantly found in plant and animal food and the deficiency is always related to deficiency of other nutrients such as calcium. symptoms include loss of appetite, joint stiffness, numbness, weakness, changes in body weight, irritability etc. phosphorus deficiency can also lead to anxiety and fatigue in the worst cases. Phosphorus rich foods are beverages like beer, chocolate or cocoa drinks, milk products such cheese and yogurt, seafood, dried fruits and whole grains, etc. However, not all phosphorus rich food is part of a healthy diet.

6. Iron: iron has several functions such as production of haemoglobin, myoglobin in muscle tissues, enzyme reactions, and maintaining a healthy immune system. Iron deficiency can be caused due to excessive blood loss, drugs interfering with absorption, malabsorption syndrome or excessive phosphorus in blood. Infants who are not breastfed could acquire iron deficiency as they require a 
high amount of iron for producing blood and muscles. Iron deficiency or hypoferremia or sideropenia leads to anaemia where haemoglobin level decreases thus decrease in red blood cells. Anaemia caused due to iron deficiency is the most common type of anaemia. The symptoms include fatigue, weakness and hair loss. Iron rich foods are pistachios, lentils, leafy vegetables and fortified cereals.(6)

7. Iodine: iodine is necessary for the proper functioning of thyroid gland. Thyroid gland controls metabolism of fat and also human embryo development. Iodine deficiency during pregnancy can cause various health defects and in adults it can cause abnormal enlargement of the thyroid gland called goitre. The causes are low dietary intake, pregnancy, increased intake of calcium, selenium deficiency, smoking tobacco, alcohol and even oral contraceptives. The major symptoms are heart failure, depression, neuropathy, miscarriage, preterm delivery and impaired ovulation which may cause infertility. In severe cases congenital iodine deficiency also known as cretinism is developed. The foods rich in iodine are seaweed, grains, egg and ionized salt.

8. Zinc: zinc is required for protein synthesis and is a component of insulin, it helps with vitamin $\mathrm{A}$ and $\mathrm{E}$ absorption and wound healing. Zinc plays a vital role in the growth and development of the human body. A regular intake of zinc is required as our body does not store it for a long time. Zinc deficiency can be caused due to liver and kidney disorders, diabetes, alcoholism, pregnancy. Vegetarians are at a higher risk of acquiring zinc deficiency as it is available in a less amount in vegetables. The deficiency is characterised by small stature, acne, cold and flu, loss of vision, lack of sexual malnutrition and impaired immunity system. The source of zinc are red meat, poultry, beans, nuts, whole grains and dairy products.

9. Selenium: selenium provides protection from oxidative damage and infection of the immune system, heart and plays a major role in reproduction and thyroid hormone metabolism. Premature infants are always born with a lower amount of selenium but it does not lead to any serious problems. Selenium deficiency is majorly observed in regions of the world where soil lacks selenium like some parts of china. The plants grown there have lower selenium content and hence a selenium deficient diet. Selenium deficiency along with other viral infections can cause cardiomyopathy and, in some cases, male sterility. Nuts, seafood, grains and poultry are rich in selenium.

10. Chlorine: chlorine deficiency is also called hypochloraemias. Chlorine takes part in several metabolic reactions and also helps in maintaining the $\mathrm{pH}$ balance of the body. Chlorine is an electrolyte hence its quantity in our body is regulated by the kidney. Electrolyte imbalance is triggered majorly by excess fluid loss, emphysema, heart failure and drugs such as laxatives, bicarbonates and diuretics. Low chlorine could eventually lead to hyponatremia, fluid loss, difficulty in breathing or weakness. The dietary sources are table salt, meat, milk, etc.

11. Manganese: manganese is an antioxidant and protects our body from oxidative damage. Manganese dependent superoxide dismutase is a detoxifier and requires manganese. It is also necessary for enzymatic reactions, wound healing and maintaining bone health. Manganese is also used in the treatment of a few cognitive diseases. The use of processed wheat flour in diet reduces the manganese intake. Diabetes, pancreatic diseases, epilepsy, haemodialysis and even the increasing amount of other minerals such as iron, phosphorus and calcium can increase the risk of acquiring this deficiency. Manganese deficiency causes schizophrenia, poor bone growth or wound healing, dermatitis, menstrual problems and low fertility. Clove, cardamom, ginger, pineapple, dark chocolates, nuts, wheat and brown rice are rich sources of manganese.

12. Copper: copper takes part in processing iron, maintaining the functioning of the nervous system and many enzymes are depended on it for function. Dietary deficiency of copper is rare and is mostly caused by secondary reasons such as malabsorption or due to surgeries. Zinc toxicity or hereditary disorders like Meke's syndrome which is an x linked recessive disorder can also cause hypocupremia or copper deficiency. Mild symptoms include poor growth of hair, pale skin and loss of control of movements. In severe cases neurological problems like neuropathy and myelopathy and haematological problems such as anaemia could develop. The dietary sources of copper are bread, cereals, nuts, sunflower seeds, potato, broccoli.

13. Chromium: chromium maintains sugar level, reduces blood pressure and also maintains brain function. Athletes, diabetes patients, pregnant women and elderly are at higher risk and chromium deficiency is caused due dehydration, increased urinary excretion and malabsorption. The symptoms are glucose intolerance, neuropathy, weight loss, high blood pressure and fluctuating cholesterol, high level of low-density lipoprotein and low level of high-density lipoprotein. Brewer's yeast, onion, apples, mushrooms, meat and whole grains are rich sources of chromium.

14. Molybdenum: molybdenum is found in beans, chickpea, lentils, dark green leafy vegetables and meat. Molybdenum absorbed from the diet is converted into cofactors and these cofactors are necessary for the functioning of some essential enzymes. It also takes part in breaking down sulphites and nitrogen metabolism. Molybdenum deficiency is a very rare condition and can be caused due to some genetic disorders. People undergoing parenteral nutrition are at high risk of acquiring this deficiency. The deficiency can cause brain abnormalities like seizures, increased level of sulphite and uric acid and also sexual problems in older males. 
15. Cobalt: cobalt helps with the production of enzymes, iron absorption and in the production of red blood cells and hormones. It is a major component of vitamin B12. The deficiency symptoms are similar to that of vitamin B12 deficiency like anaemia or impaired nervous system. The main source of cobalt is green leafy vegetables. If the deficiency is prolonged it could lead to severe changes in the body that are irreversible.

16. Fluoride: fluoride is important for the teeth and bones. It protects the teeth and also stimulates the formation of bones. Most food contains fluoride in very less amount but marine fishes, tea mainly decaffeinated teas contains comparatively high amounts. Increased concentration of calcium can reduce the absorption of fluoride. Its deficiency majorly affects the teeth and bones. Most of the dental products contain fluoride and thus supply fluoride to the body.

17. Sulphate: sulphate is a vital component of amino acids like methionine and cysteine. It protects the protoplasm of cells as it is part of antioxidants and also help in cleansing of the blood. Sulphur is present in all the food sources that contain proteins. The body receives sulphate from body protein turnover. The sources of sulphate are dried fruits, soy, etc. sulphate deficiency causes obesity, heart diseases and stress.

There are other minerals like arsenic, nickel, boron and vanadium which are part of our diets but no evident dietary deficiency complications are observed.

\section{TREATMENT AND PREVENTION NUTRITIONAL DEFICIENCY DISEASES}

OF

Nutritional deficiency diseases are diagnosed through the study of the symptoms and blood test like in case of most minerals.(7)

\section{A. TREATMENT}

i. Adequate intake (AI) is the estimated quantity of a specific nutrient required by a human body. It is important to know the adequate intake level of any nutrient before starting the treatment. Despite being vital, the nutrients are toxic if consumed in large amount and also the quantity of proteins, calories, vitamins and minerals a person should consume depends on their age, sex and their physical activity level and other secondary reasons. When the deficiency is caused due to secondary reasons then the treatment for the underlying medical conditions is given first.

ii. Treatment of nutritional deficiency takes place in the following modes: iii. Dietary changes - the patient is given a healthy and balanced diet. Multivitamins and supplements are added according to the need of the patient. Fortified food is also used commonly.

iv. Parenterally or intravenously - this method is opted mostly when the patient is suffering from chronic deficiency.

$\mathrm{v}$. The treatment of protein energy malnutrition depends upon the severity of the disease. Treatments can lead to refeeding symptoms where the level of other nutrients increases such as hypophosphatemia, hypokalemia and hypomagnesemia that disrupts the organ systems and their functions. The treatment takes place in different phases. The main aim of the treatment is stabilisation that is to restore normal body composition treating infection, correcting fluids and electrolyte imbalance, anaemia and other nutrient deficiencies. Strategies are adopted to prevent hypothermia. Then administering Antibiotics that does not affect protein synthesis and then gradual increase in dietary proteins. As marasmus and kwashiorkor causes a deep impact on the cognitive system, mental health treatment and support is also recommended in the later phases of the treatment.

vi. Vitamin deficiency can be treated successfully by supplementation and dietary changes.

\section{B. PREVENTION}

Nutritional deficiency can be prevented successfully. Providing health education and making people aware of the nutritional requirements of their own bodies is one of the most important methods. The major preventive measures are:

Increasing breast feeding - breast milk contains all the nutrition that a child need. It strengthens the immune system and helps in proper growth and development of the child. It even protects the child from many adulthood diseases. Breast fed children are at very less risk of acquiring protein energy malnutrition.

i. Promoting immunisation and healthy feeding practise for infants and children

ii. Ensuring older people receive an optimal supply of the nutrients. if in old age the body does not receive the sufficient amount of vitamin, minerals, fatty acids, etc. the aging process will increase thus impairing cell renewal and immune system

iii. Eating a healthy variety of food and including healthy fats and omega 3

iv. Drinking healthy beverages low in processed sugar

v. Following an active lifestyle

vi. Eating food rich in fibres and antioxidants

vii. Reducing the intake of processed food- processed food mainly contains very less amount of nutrients and all they 
contribute is by increasing the calorie intake and causing health problems.

viii. Genetic engineering methods like biofortification can be used to enhance the quality and quantity of nutrients in food crops. Example: golden rice which has high content of vitamin A and other crops like cassava and sweet potato. Iron fortification of wheat flour, iodine fortification of salt, zinc fortification of plant-based food and folic acid fortification of cereal grains have shown exceptional results.

ix. Government policies and campaigns that provide supplements and healthy food at an affordable rate to vulnerable groups.

\section{CONCLUSION}

Human body needs the right amount of nutrients to function at its true potential. Deficiency of one nutrient can even lead to severe complications and increased levels of one nutrient can cause deficiency of another nutrient and vice versa. Nutritional deficiency is most common among pregnant and lactating women, children and adolescents who need high amounts of nutrients for their growth and development and also elderly and people who are critically ill as they may suffer malabsorption. Maximum of these deficiencies can be overcome with suitable treatment but in cases where the deficiency is very severe it could lead to irreversible changes hence deficiencies should be treated in its early stages. Undernutrition can lead to a variety of acute and chronic complications that harms the growth and development of the body. Low health literacy is one of the major causes of nutritional deficiency and also poverty which keeps people away from healthy and balanced diets. In many parts of the globe right strategies and aid from the authorities has helped in tackling these problems. Strategies for dietary diversification has to be opted as isolated cases of nutrient deficiencies are less. The prevalence of nutritional deficiencies has declined in some parts of the world but where hunger and poverty are the biggest problem chronic undernourishment still exists.

\section{REFERENCE}

1) https://www.who.int/elena/nutrient/en/

2) Samuel

Klein,Protein-Energy Malnutrition,Goldman's Cecil Medicine (Twenty Fourth Edition),2012,Pages 1388-1391, https://www.sciencedirect.com/topics/medicine-anddentistry/protein-calorie-malnutrition

3) Weininger J. Nutritional disease [Internet]. Encyclopædia Britannica. Encyclopædia Britannica, inc.; 2020 [cited 2020Apr15]. Available from: https://www.britannica.com/science/nutritionaldisease
4) World Health Organisation, Food and agricultural organisation of united nations. Vitamin and mineral requirements in human nutrition, (2004)https://www.who.int/nutrition/publications/mic ronutrients/9241546123/en/

5) Institute of medicine, Dietary reference intake: the essential guide to nutrient requirements, The National academics press Washington DC,2006) https://books.google.co.in/books?id=dYZZTgjDeccC $\& p g=$ PA $82 \&$ source $=$ gbs_toc_r $\&$ cad $=3 \# \mathrm{v}=$ onepage $\&$ $\mathrm{q} \& \mathrm{f}=$ false

6) Ritchie H, Roser M. Micronutrient Deficiency [Internet]. Our World in Data. 2017 [cited 2020Apr28]. Available from: https://ourworldindata.org/micronutrient-deficiency

7) Nutritional Deficiencies (Malnutrition): Symptoms and ... [Internet]. [cited 2020May1]. Available from: https://www.healthline.com/health/malnutrition

8) World Health Organisation (2003): Diet, nutrition, and the prevention of chronic disease. Report of a WHO/FAO study group, Technical Report Series, No. 916, WHO, Genevahttps://www.who.int/dietphysicalactivity/publ ications/trs916/en/

9) Micronutrient Malnutrition Prevention and Control [Internet]. Centers for Disease Control and Prevention. Centers for Disease Control and Prevention; 2015 [cited 2020May7]. Available from: https://www.cdc.gov/nutrition/micronutrientmalnutrition/index.html 